Anna Rowe 3,5 , Philip N. Newsome ${ }^{3,6}$, Jessica Douglas-Pugh (D) ${ }^{5}$,

Sheeba Khan ${ }^{3,6,7}$, Saloni Mittal ${ }^{8}$,

Roger Wilson (D) ${ }^{9}$, Daniel O'Connor ${ }^{10}$, Lisa Campbell ${ }^{10}$, Sandra A. Mitchell ${ }^{11}$ and Melanie Calvert (D) 1,2,3,4,12,13凶

${ }^{1}$ Centre for Patient Reported Outcomes Research, Institute of Applied Health Research, University of Birmingham, Birmingham, UK. ${ }^{2}$ National Institute for Health Research Applied Research Centre West Midlands, University of Birmingham, Birmingham, UK. ${ }^{3}$ National Institute for Health Research Birmingham Biomedical Research Centre at University Hospitals Birmingham NHS Foundation Trust, Birmingham, UK. ${ }^{4}$ Birmingham Health Partners Centre for Regulatory Science and Innovation, Birmingham, UK. ${ }^{5}$ Cancer Research UK Clinical Trials Unit, University of Birmingham, Birmingham, UK. ${ }^{6}$ Centre for Liver and Gastrointestinal Research, Institute of Immunology and Immunotherapy, University of Birmingham, Birmingham, UK. ${ }^{7}$ Liver Unit, University Hospitals Birmingham NHS Foundation Trust, Birmingham, UK. ${ }^{8}$ University of Birmingham, Birmingham, UK. ${ }^{9}$ NCRI Consumer Forum National Cancer Research Institute, London, UK. ${ }^{10}$ Medicines and Healthcare Products Regulatory Agency (MHRA), London, UK. ${ }^{11}$ Outcomes Research Branch, Division of Cancer Control and Population Sciences, National Cancer Institute, Rockville, MD, USA. ${ }^{12}$ National Institute for Health Research Surgical Reconstruction and Microbiology Research Centre, University of Birmingham, Birmingham, UK. ${ }^{13}$ UK SPINE, University of Birmingham, Birmingham, UK.

$\bigotimes_{e-m a i l: m . c a l v e r t @ b h a m . a c . u k}$

Published online: 17 January 2022

https://doi.org/10.1038/s41591-021-01648-4
References

1. US Food and Drug Administration (FDA). The Drug Development Process-Step 3: Clinical Research; https://www.fda.gov/patients/ drug-development-process/step-3-clinical-research (FDA, 2018).

2. Coleman, R. L. et al. Oncology 99, 444-453 (2021).

3. Basch, E. \& Dueck, A. C. Exp. Opin. Drug Discov. 11, 753-758 (2016).

4. Fiteni, F. et al. BMC Cancer 19, 361 (2019).

5. Kirkham, J. J. et al. PLoS Med. 14, e1002447 (2017).

6. Calvert, M. et al. JAMA 319, 483-494 (2018).

7. Trotti, A. et al. Semin. Radiation Oncol. 13, 176-181 (2003).

8. Basch, E. et al. J. Natl Cancer Inst. 101, 1624-1632 (2009).

9. Kluetz, P. G. et al. Am. Soc. Clin. Oncol. Educ. Book 35, 67-73 (2016).

10. Chen, A. C., Mitchell, S. A., Minasian, L. M. \& St. Germain, D. in Novel Designs of Early Phase Trials for Cancer Therapeutics (ed. Takimoto, K. A.) 193-208 (Elsevier, 2018). 11. Dueck, A. C. et al. JAMA Oncol. 1, 1051-1059 (2015). 12. Trask, P. C. et al. Clin. Trials 15, 616-623 (2018).

13. Shepshelovich, D. et al. Oncologist 24, e146-e148 (2019).

14. Pearman, T. P. et al. Cancer 124, 991-997 (2018).

15. FDA. Core Patient-Reported Outcomes in Cancer Clinical Trials: Draft Guidance for Industry (June 2020 draft); https://www.fda. gov/regulatory-information/search-fda-guidance-documents/ core-patient-reported-outcomes-cancer-clinical-trials (FDA, 2021).

16. Chalasani, M., Vaidya, P. \& Mullin, T. Res. Involve. Engagement 4, 10 (2018).

17. FDA. Guidance for Industry: Patient-Reported Outcome Measures: Use in Medical Product Development to Support Labeling Claims; https://www.fda.gov/ regulatory-information/search-fda-guidance-documents/ patient-reported-outcome-measures-use-medicalproduct-development-support-labeling-claims (FDA, 2009). 18. Proceedings of Patient Reported Outcome Measures (PROMs) Conference Birmingham 2018. J. Patient Rep. Outcomes 2, 58 (2018).

19. UK Medicines and Healthcare Products Regulatory Agency. Innovative Licensing and Access Pathway https://www.gov.uk/ guidance/innovative-licensing-and-access-pathway (accessed 13 December 2021)

20. Kluetz, P. G., O'Connor, D. J. \& Soltys, K. Lancet Oncol. 19, e267-e274 (2018)

21. Kluetz, P. G. et al. Value Health 21, 742-747 (2018).

22. Tavridou, A. et al. Br. J. Clin. Pharmacol. 87, 2459-2464 (2021).

23. West, H. JAMA Oncol. 3, 423-423 (2017).

\section{Acknowledgements}

We thank A. Filer, P. Trivedi, D. Chanouzas, S. Ghosh and A. Dueck for their advice and suggestions during this project. The author(s) disclose receipt of the following financial support for the research, authorship and/or publication of this article: This work was supported by the National Institute for Health Research (NIHR) Birmingham Biomedical Research Centre, Midlands-Wales Advanced Therapies Treatment Centre (MW-ATTC) program grant from Innovate UK to a consortium of partners including Health Technology Wales, the Welsh Blood Service and the University of Birmingham (grant number: IUK: 104232) and Innovate UK (part of UK Research and Innovation) grant Patient-reported outcomes assessment to support accelerated access to advanced cell and gene therapies: PROmics (grant number: 104777). The funder was not involved in any aspect of the research work. O.L.A., A.R. and M.C. receive funding from the NIHR Applied Research Collaboration, West Midlands (ARC), and the Brain Tumour Charity. O.L.A, receives funding from the NIHR Birmingham Biomedical Research Centre (BRC, West Midlands at the University of Birmingham) and University Hospitals Birmingham NHS Foundation, Innovate UK (part of UK Research and Innovation), Gilead Sciences Ltd. and Janssen Pharmaceuticals Inc. MC is Director of the Birmingham Health Partners Centre for Regulatory Science and Innovation, Director of the Centre for Patient Reported Outcomes Research and is a National Institute for Health Research (NIHR) Senior Investigator. She receives funding from the NIHR, UK Research and Innovation (UKRI), NIHR Birmingham Biomedical Research Centre, the NIHR Surgical Reconstruction and Microbiology Research Centre and UK SPINE at the University of Birmingham and University Hospitals Birmingham NHS Foundation Trust, Health Data Research UK, Innovate UK (part of UKRI), Macmillan Cancer Support, UCB, Gilead, Janssen and GSK Pharma. The views expressed in this article are those of the authors and not necessarily those of the NIHR, the Department of Health and Social Care or the MHRA.

\section{Competing interests}

O.L.A. declares personal fees from Gilead Sciences Ltd., GlaxoSmithKline (GSK) and Merck outside the submitted work. M.C. has received personal fees from Astellas, Aparito Ltd., CIS Oncology, Takeda, Merck, Daiichi Sankyo, Glaukos, GSK and the Patient-Centered Outcomes Research Institute (PCORI) outside the submitted work. The other authors declare no competing interests.

\title{
Post-acute neurological consequences of COVID-19: an unequal burden
}

COVID-19 and its neurological consequences particularly burden marginalized communities, and so can only be effectively treated by advancing health equity.

\section{LaShyra T. Nolen, Shibani S. Mukerji and Nicte I. Mejia}

O ur world has witnessed over 275 million confirmed cases of COVID19 and over 5 million related deaths ${ }^{1}$. Marginalized communities everywhere continue to be disproportionately affected as the pandemic amplifies longstanding health and healthcare disparities. As an example, in the United States, members of the Black, Indigenous and Latino communities remain two to three times more likely to be infected with SARS-CoV-2, to be hospitalized with COVID-19 and to die from this disease ${ }^{2}$. Dismantling structural racism is necessary to improve neurological health, as greater attention is focused on understanding and addressing the post-acute neurological consequences of COVID-19, or the neurological 


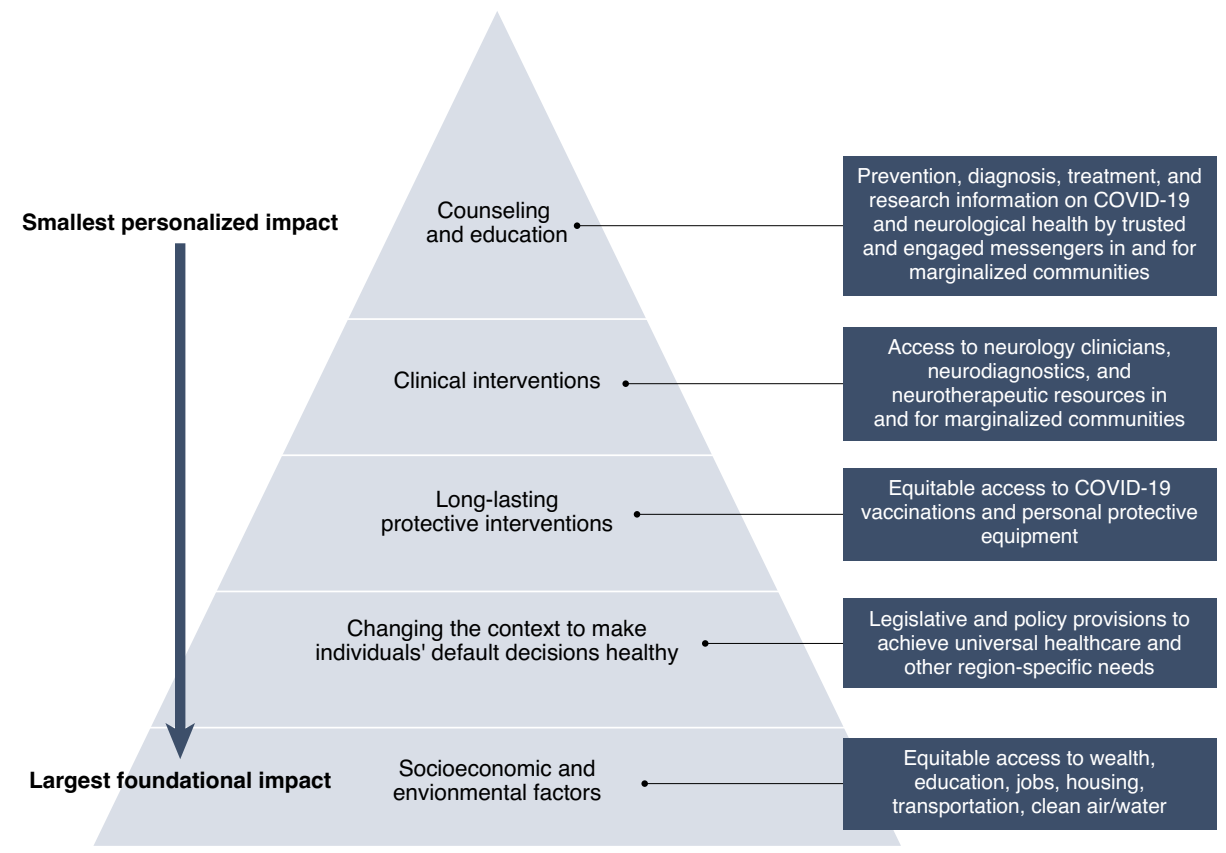

Fig. 1 | Framework to advance health equity in neurology. The proposed model is adapted from the United States CDC Health Impact Pyramid ${ }^{14}$.

manifestations of what is sometimes called long COVID.

\section{Neurological problems}

The prevalence of neurological problems associated with COVID-19 in the acute and subacute phases of illness is 35-85\% $(\text { Table } 1)^{3-5}$. People commonly report cognitive or memory disturbances, headache, loss of smell or taste, and myalgia. Acute neurological diagnoses include encephalopathy, delirium, cerebrovascular disease, seizures, neuropathy and myopathy. Less frequently reported problems include abnormal movements, psychomotor agitation, syncope and autonomic dysfunction. Para-infectious complications, such as acute demyelinating encephalomyelitis, acute necrotizing encephalopathy, acute inflammatory demyelinating polyneuropathy and autoantibody-suspected neurological manifestations, have been documented in small retrospective studies, but data regarding their prevalence remain inadequate.

Knowledge of the post-acute neurological consequences of COVID-19 is still very limited. Among the largely American and European respondents to an online survey available in nine languages ${ }^{6}$, a substantial proportion of those who had had COVID-19 endorsed neurological problems that persisted 3-6 months after diagnosis. Symptoms included memory or cognitive disturbances, post-exertional malaise or fatigue, insomnia and other sleep disturbances, headache and loss of smell or taste. Approximately $30 \%$ of those aged 30-59 who reported cognitive disturbance felt severely unable to function at work. Although some respondents indicated that their neurological symptoms gradually improved over time, others described a disturbing pattern of relapse and remittance, noting triggers such as physical activity, mental exertion, emotional stress and menstruation. Among the respondents who had tested positive for SARS-CoV-2 but not been hospitalized, neurological problems that persisted at 3-9 months from their date of infection included vertigo, depression, memory and cognitive disturbances, and taste or smell disturbances; many recipients of new neurological diagnoses were younger than 65 years $^{7}$.

\section{Unrepresentative research}

The pervasive segregation of resources and power perpetuates structural barriers that limit our understanding of the true breadth of neurological problems associated with COVID-19. Although inequities in access to SARS-CoV-2 testing persist, most research stems from people with a positive SARS-CoV-2 polymerase chain reaction (PCR) test, and few studies consider people whose COVID-19 diagnoses were based on symptoms or an antibody response. There is heavy reliance on mining electronic health records using billing diagnostic codes, which requires digital infrastructure and scientific expertise often found at large academic medical institutions in high-income nations. Few studies engage with people, and if so, engagement is often through surveys that tend to rely on digital technology and exist in a few languages, such as English. It is rare for studies to include in-person clinical evaluations. Participation in research beyond Europe and the United States remains very limited, and even within those geographic regions, marginalized communities are significantly underrepresented despite their disproportionate COVID-19 burden.

To fully understand the neurological complications of long COVID, there must be more equity in COVID-19 research, which in turn requires a dismantling of structural barriers that perpetuate disparities in clinical care. It is clear that inequities in access to hospital resources are pathways to neurological disparities related to acute COVID-198. A retrospective analysis of people hospitalized with COVID-19 and stroke across the United States and Canada found that subpar access to acute stroke treatments such as thrombolysis and thrombectomy contributed to Black patients being twice as likely to die as patients of other races or ethnic backgrounds, despite having similar stroke risk factors and evaluation times ${ }^{9}$. Another retrospective analysis of patients hospitalized with COVID-19 across a large American healthcare system documented how receiving care at non-academic hospitals posed double the risk of 30-day mortality and worse functional outcomes, despite patients having similar COVID-19 severity and fewer comorbidities, presumably because of less access to critical care and other specialty services ${ }^{10}$.

There is growing documentation of healthcare disparities faced by people who have had COVID-19, including those with and without neurological complications. Among people surveyed after discharge from 38 Michigan hospitals for the treatment of acute COVID-19, one in five had not received follow-up care within 60 days of hospital discharge, with $60 \%$ of the patients who received no care identifying as Black and $5 \%$ as Latino ${ }^{11}$. The same study showed that people of color who had COVID-19 were most likely to report lack of health insurance and moderate to severe financial effects, with the majority using up their savings and being unable to cover the cost of health-related supplies. Alongside this, a qualitative study of the experiences of Latinos hospitalized for treatment of acute COVID-19 in San Francisco and Denver described some 


\section{Table 1 | Nervous system problems observed during the acute, para-infectious and/or post-acute phases of COVID-19}

\begin{tabular}{|c|c|c|c|}
\hline & Acute & Post-acute & Para-infectious \\
\hline \multicolumn{4}{|l|}{ Cerebrovascular } \\
\hline Ischemic stroke & $x$ & $x$ & \\
\hline Hemorrhagic stroke & $x$ & $x$ & \\
\hline Venous sinus thrombosis & $x$ & $x$ & \\
\hline \multicolumn{4}{|l|}{ Chemosensory } \\
\hline Smell disturbance & $x$ & $x$ & \\
\hline Taste disturbance & $x$ & $x$ & \\
\hline \multicolumn{4}{|l|}{ Cognitive and memory } \\
\hline Coma & $x$ & & \\
\hline Delirium & $x$ & & \\
\hline Encephalopathy & $x$ & $x$ & \\
\hline \multicolumn{4}{|l|}{ Mood } \\
\hline Anxiety & $x$ & $x$ & \\
\hline Depression & & $x$ & \\
\hline Post-traumatic stress disorder & & $x$ & \\
\hline \multicolumn{4}{|l|}{ Neuromuscular } \\
\hline Acute inflammatory demyelinating polyneuropathy & & & $x$ \\
\hline Myalgias & $x$ & $x$ & \\
\hline Myopathy & $x$ & $x$ & \\
\hline Neuropathy & $x$ & $x$ & \\
\hline Weakness & $x$ & $x$ & \\
\hline \multicolumn{4}{|l|}{ Other } \\
\hline Abnormal movements & $x$ & $x$ & \\
\hline Acute necrotizing encephalitis & & & $x$ \\
\hline Acute disseminated encephalomyelitis & & & $x$ \\
\hline Auto-antibody-mediated disorders & & & $x$ \\
\hline Dizziness & $x$ & $x$ & \\
\hline Dysautonomia & $x$ & $x$ & \\
\hline Fatigue & $x$ & $x$ & \\
\hline Headache & $x$ & $x$ & \\
\hline Meningoencephalitis & $x$ & & \\
\hline Multisystem inflammatory syndrome & & & $x$ \\
\hline Sleep disturbances & $x$ & $x$ & \\
\hline Seizures & $x$ & & \\
\hline
\end{tabular}

participants being discharged home without follow-up treatments such as oxygen or physical therapy because they lacked health insurance benefits ${ }^{12}$. These early gaps in access to post-acute COVID-19 services are likely the tip of the iceberg and threaten to negatively affect the neurological health of individuals with COVID-19, potentially long term.

\section{Community-centered health}

An anti-racist, patient- and community-centered approach must be adopted to identify, comprehensively understand and effectively treat the post-acute consequences of COVID-19, including neurological sequelae. This requires shifting meaningful resources and power to marginalized communities most affected by COVID-19, while effectively health action (Fig. 1). Although all the interventions in the proposed model are important to advance health equity, greater and longer-lasting impact will be achieved in moving from the individual to the societal factors that influence health disparities ${ }^{13}$. The neurology community needs to collaborate applying a multi-level framework for public across sectors to amplify the impact of work to dismantle structural racism. Interdisciplinary policy solutions beyond medicine, public health and government are critical to quell the spread of SARS-CoV-2, decrease mortality and ultimately prevent the debilitating neurological and other burdens of COVID-19.

Underserved communities need access to trusted messengers who can provide quality information about neurological health as well as effective public health interventions to decrease the risk of COVID-19, such as physical distancing, mask wearing and regular hand-washing ${ }^{14}$. Access to trusted messengers should be complemented by access to high-quality neurological services within and for marginalized communities. This access is particularly important for people of color and those experiencing poverty, as these marginalized communities have been left out of neurological care from before the COVID-19 pandemic ${ }^{15}$. Safety-net hospitals, community-based health centers and other resource-limited clinical settings must be well equipped to offer expansive screening of neurological problems, provide in-person or telehealth-assisted neurological services, and have processes to refer patients to other medical institutions for higher-level care when needed.

Wider access to neurology clinicians will be most impactful if accompanied by access to high-quality diagnostic modalities such as brain imaging, electroencephalography, electromyography, sleep studies and neuropsychological testing. Access to diagnostics should be coupled with increased access to potentially life-changing therapeutics both for COVID-19 and for its neurological sequelae, ranging from vaccines, monoclonal antibodies, thrombolysis, thrombectomy and rehabilitation services including physical, occupational and cognitive therapy, to innovations such as the new oral antiviral drugs molnupiravir (Lagevrio; Merck) and a combination of nirmatrelvir and ritonavir (Paxlovid; Pfizer). Vaccines are especially important in preventing COVID-19 and its long-term neurological sequelae, and marginalized communities continue to face a complex web of structural barriers to vaccination.

\section{Comprehensive care}

Appropriate healthcare insurance coverage will eliminate an important barrier to access to neurological services ${ }^{16,17}$. In the United States alone, most of the 31 million uninsured adults forego diagnostic and treatment opportunities because of financial concerns. Universal healthcare has been proposed by the World Health 
Organization to allow people to have access to the health services that they need without financial hardship, and should be supported by the neurology community, whose members can learn about and engage in efforts that define a path to universal healthcare ${ }^{17-19}$.

In countries without universal healthcare, there is an urgent need for medical care and support services for patients with COVID19 , many of whom face financial barriers to healthcare. The US government has allocated government funds toward COVID19 testing and vaccination efforts, but there is a gap in funding for post-acute COVID19 clinical services, including physical rehabilitation, mental health, home health, long-term care and other health services often not covered by health insurance. A successful example of a comprehensive model of care is the United States' Ryan White HIV/AIDS Program, a discretionary measure created three decades ago in the face of another public health crisis ${ }^{20}$. The Ryan White Program collaborates with a broad array of stakeholders at the federal, state and local levels, including community-based organizations, working together to implement public health interventions that aim to prevent and reduce transmission of HIV, improve clinical care and achieve positive health outcomes, while addressing the disproportionate impact of
HIV on the Black, Latino, Indigenous and uninsured communities.

Black, Latino and Indigenous people are overrepresented as essential workers, with low-paying jobs, lack of adequate benefits such as paid leave and limited access to personal protective equipment, all of which contribute to a disproportionate impact from COVID-19, including its neurological sequelae. Research to understand the acute and post-acute neurological consequences of COVID-19 should be centered in the experiences and lives of people. Patient-centered research will require a commitment to dismantling the systemic forms of oppression that have led to disparate suffering among marginalized communities well before this pandemic. This starts with naming racism and engaging in anti-racist solutions to achieve health justice in and beyond neurology, including increasing access to quality neurological evaluations, treatments and research options. Prioritizing equity today will improve the delivery of neurological care far beyond this pandemic.

LaShyra T. Nolen', Shibani S. Mukerji (D) 1,2 and Nicte I. Mejia (D) 1,2凶

${ }^{1}$ Harvard Medical School, Boston, MA, USA.

${ }^{2}$ Department of Neurology, Massachusetts General Hospital, Boston, MA, USA.

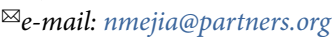

Published online: 17 January 2022

https://doi.org/10.1038/s41591-021-01647-5

References

1. Dong, E. et al. Lancet Infect. Dis. 20, 533-534 (2020).

2. Centers for Disease Control and Prevention-National Center for Health Statistics. https://www.cdc.gov/nchs/nvss/vsrr/covid19/ health_disparities.htm (accessed November 2021).

3. Chou, S. H. Y. et al. JAMA Netw. Open 4, e2112131 (2021).

4. Ross Russell, A. L. et al. Brain Commun. 3, fcab168 (2021).

5. Mao, L. et al. JAMA Neurol. 77, 1 (2020).

6. Davis, H. E. et al. EClinicalMedicine 38, 101019 (2021).

7. Estiri, H. et al. BMC Med. 19, 249 (2021).

8. Nolen, L. \& Mejia, N. I. Nat. Rev. Neurol. 17, 67-68 (2021).

9. de Havenon, A. et al. JAMA Netw. Open 4, e2110314 (2021).

10. Dmytriw, A. A. et al. J. Neurol. Neurosurg. Psychiatry 91, 1362-1364 (2020).

11. Robinson-Lane, S. G. et al. J. Am. Med. Dir. Assoc. 22, 2245-2250 (2021).

12. Cervantes, L. et al. JAMA Netw. Open 4, e210684 (2021).

13. Frieden, T. R. N. Engl. J. Med. 373, 1748-1754 (2015).

14. Alsan, M. et al. Ann. Intern. Med. 174, 484-492 (2021).

15. Saadi, A. et al. Neurology 88, 2268-2275 (2017).

16. Kaiser Family Foundation. https://www.kff.org/uninsured/ (accessed November 2021).

17. World Health Organization. https://www.who.int/health-topics/ universal-health-coverage (accessed November 2021).

18. Verguet, S. et al. Nat. Med. 27, 380-387 (2021).

19. Editorial. Nature 593, 313-314 (2021).

20. Mandsager, P. et al. Am. J. Public Health 108, S246-S250 (2018).

\section{Author contributions}

Lead author duties were performed by N.I.M., with all the authors contributing equally to the conceptual framework, drafting, critical review and finalization of the manuscript.

Competing interests

The authors declare no competing interests. 